\title{
Topology optimization of coated structures and material interface problems
}

\author{
Clausen, Anders; Aage, Niels; Sigmund, Ole
}

Published in:

Computer Methods in Applied Mechanics and Engineering

Link to article, DOI:

10.1016/j.cma.2015.02.011

Publication date:

2015

Document Version

Peer reviewed version

Link back to DTU Orbit

Citation (APA):

Clausen, A., Aage, N., \& Sigmund, O. (2015). Topology optimization of coated structures and material interface problems. Computer Methods in Applied Mechanics and Engineering, 290, 524-541.

https://doi.org/10.1016/j.cma.2015.02.011

\section{General rights}

Copyright and moral rights for the publications made accessible in the public portal are retained by the authors and/or other copyright owners and it is a condition of accessing publications that users recognise and abide by the legal requirements associated with these rights.

- Users may download and print one copy of any publication from the public portal for the purpose of private study or research.

- You may not further distribute the material or use it for any profit-making activity or commercial gain

- You may freely distribute the URL identifying the publication in the public portal

If you believe that this document breaches copyright please contact us providing details, and we will remove access to the work immediately and investigate your claim. 


\title{
Topology optimization of coated structures and material interface problems
}

\author{
Anders Clausen $^{\mathrm{a}, *}$, Niels Aage ${ }^{\mathrm{a}}$, Ole Sigmund ${ }^{\mathrm{a}}$ \\ ${ }^{a}$ Department of Mechanical Engineering, Solid Mechanics, Technical University of Denmark, \\ Nils Koppels Alle, B. 404, DK-2800 Lyngby, Denmark
}

\begin{abstract}
This paper presents a novel method for including coated structures and prescribed material interface properties into the minimum compliance topology optimization problem. Several elements of the method are applicable to a broader range of interface problems. The approach extends the standard SIMP method by including the normalized norm of the spatial gradient of the design field into the material interpolation function, enforcing coating material at interfaces by attributing particular properties. The length scales of the base structure and the coating are separated by introducing a two-step filtering/projection approach. The modeled coating thickness is derived analytically, and the coating is shown to be accurately controlled and applied in a highly uniform manner over the structure. An alternative interpretation of the model is to perform single-material design for additive manufacturing. Infill is assumed to be constituted of an isotropic porous microstructure satisfying the Hashin-Shtrikman bounds and is modeled using the homogenized material properties. A range of numerical results illustrate the effectiveness of the approach.
\end{abstract}

Keywords: Topology optimization, Coating, Interface representation, Two-step filtering, Additive manufacturing

\section{Introduction}

The objective of this study is to design coated structures by topology optimization. Metal coating of polymer structures is commonly used to enhance functional

\footnotetext{
${ }^{*}$ Corresponding author

Email address: andcla@mek.dtu.dk (Anders Clausen)
} 
or visual properties or to reduce component cost. Furthermore, many polymers are easily processed into complex shapes that metals alone could not form. Such structures, when coated with metal, combine the processing and cost advantages of polymers with the performance benefits of metal.

While there exists a wide range of metal coating technologies (Møller and Nielsen (2013)), one of the most common techniques is electroplating. In electroplating, the polymer base structure is initially etched to create small pores in the surface where the coating material can anchor. The polymer surface is then activated with palladium salts and subsequently immersed in a concentrated electroless copper or nickel plating bath for autocatalytic plating. The surface thereby becomes conductive and may be coated with a range of different metals by means of electrolytic plating. The most common polymer material used as a substrate for electroplating is acrylonitrile-butadiene-styrene (ABS). The initial etching removes the butadiene phase which allows for a very strong adhesion between polymer and coating. ABS is also widely used in additive manufacturing (AM), specifically in fused deposition modeling (FDM). Combining the advantages of metal coated polymers with the design freedom afforded by AM has the potential to create new types of architectures (Schaedler et al. (2011)).

This work models coated structures. The method assumes perfect bonding between the substrate and the coating material. The study is limited to 2D applications. Assuming the absence of closed cavities, the model should be readily expandable from $2 \mathrm{D}$ shapes to $3 \mathrm{D}$ objects.

The paper considers minimum compliance problems. The approach suggested in the paper draws on the basic ideas of the SIMP approach (for the fundamentals, see e.g. Bendsøe and Sigmund (2003)). The usual stiffness interpolation from SIMP is extended to include spatial gradients of the (filtered) design field. This allows to identify material interfaces and enforce coating. In order to control the spatial gradient field and thereby assure a uniform coating thickness a two-step filtering approach is introduced.

Spatial density gradients have earlier been used for identifying material interfaces. The context has been restriction methods / perimeter control rather than modeling of interface properties. Petersson and Sigmund (1998) introduce a slope constraint with pointwise bounds on the density slopes to ensure existence of solutions. The topic is discussed in more detail by Borrvall (2001).

Filter processes performed over several steps to control different geometric properties simultaneously have earlier been used. Sigmund (2007) provides an overview of the most common filters and shows how morphological operators may be combined to assure that several properties are simultaneously attained. 
As an example the combined open-close or close-open operator allows to obtain minimum hole size and minimum structural detail size simultaneously.

The ability to accurately describe material interfaces is often mentioned as an advantage of level-set based approaches, for which interfaces are implicitly defined by iso-contours of a level-set function. For a review of level-set based topology optimization, see e.g. van Dijk et al. (2013). Based on the level-set method, Vermaak et al. (2014) present a method for including material interface properties in the optimization of multi-phase elastic and thermoelastic structures. Rather than modeling a sharp interface between two different materials, a transition zone is introduced to allow for graded properties.

The method presented in this paper shows that a level set based method is not the only possible approach when solving problems requiring the ability to accurately describe material interfaces. A density based approach may as well be used. More generally speaking, issues which were earlier considered a drawback of density based approaches compared to level set based approaches have been solved in recent work. An example is the presence of intermediate densities in the final design. By introducing projections methods such as robust optimization (Wang et al. (2011)) this issue may to a large extent be overcome. Combined with the advantages of density based approaches (such as the availability of sensitivity information in the entire design domain), a density based approach was considered the preferred choice for solving the problem addressed in this paper. For a detailed comparison of density based vs. level set based approaches see Sigmund and Maute (2013).

The remainder of the paper is structured as follows: Section 2 presents the problem formulation. This includes the material model and the interpolation scheme assuring coating at all surfaces, as well as the optimization problem and the sensitivity analysis. Section 3 presents and discusses numerical results. A general discussion is presented in section 4 . Section 5 concludes the paper.

\section{Problem formulation}

In this section the optimization problem is defined. This includes defining an appropriate material model, formally defining the optimization problem, and deriving sensitivities. The material model and characteristic properties are derived analytically based on continuous versions of the design field and filters. Only when formally defining the optimization problem, the discretized version is introduced. 


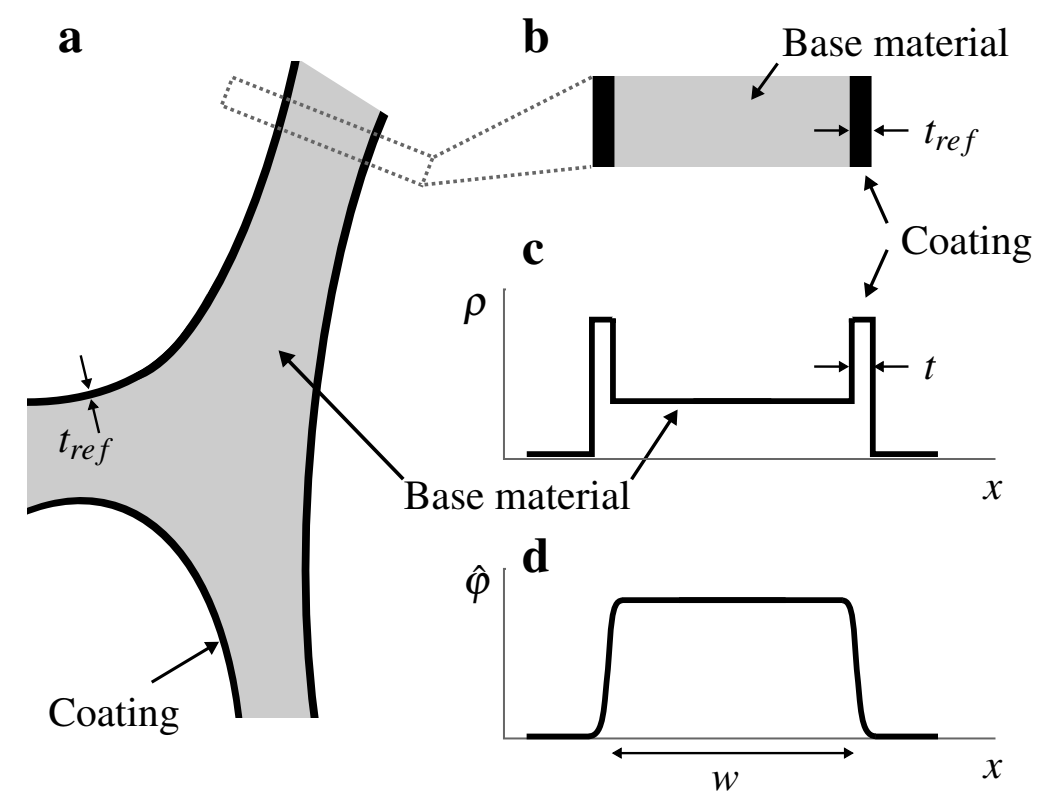

Figure 1: Illustrative sketch of relation between coated structure, filtered design field and physical density function. a Extract of a coated structure in 2D. b Feature from (a). c 1D physical density function representing the feature. d Underlying (filtered) design field with a feature of width $w$.

\subsection{Material model}

A coated structure is characterized by a base structure made of one material (referred to as the substrate in a process context) and a coating made of a different material. Initially, no limitations are put on the shape and dimensions of the base structure, whereas the coating is assumed to have a constant, pre-defined thickness, $t_{\text {ref }}$, at all surfaces of the base structure. Fig. 1 shows a sketch of a coated structure in 2D and illustrates how the associated physical density function should look like for a $1 \mathrm{D}$ cross section.

This physical density function may be obtained from a filtered $0 / 1$ design field by utilizing that the interface is characterized by a large spatial gradient, see Fig. 11. If the base material is chosen where the design field equals 1 and the coating is enforced where the spatial gradient is large, the physical density function from Fig. 1c is obtained.

It is important to notice that the prescribed coating thickness, $t_{r e f}$, is a fixed parameter which is defined as part of the design problem. For the optimization problem it is convenient to define the coating based on the spatial gradient of the design field in order to assure sufficient design freedom. The modeled coating may be characterized by a thickness, $t$. This parameter, referred to as the modeled 


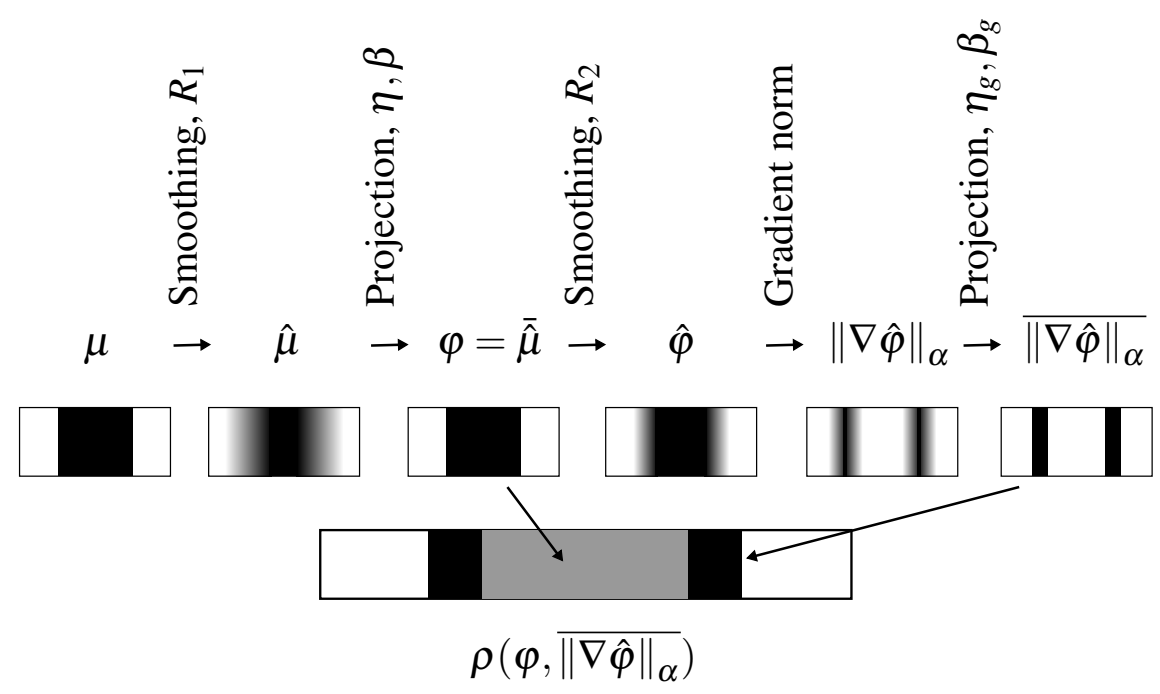

Figure 2: Two-step filter process allowing to separate length scales of base structure and coating. The physical density is an interpolation of $\varphi$ and $\overline{\|\nabla \hat{\varphi}\|_{\alpha}}$.

coating thickness, therefore depends on the design field. This approach assures sufficient design freedom, but must be combined with a method to control the gradient field in order to end up with the correct coating thickness in the final design.

To control the shape of the gradient a two-step filtering process is applied as illustrated in Fig. 2, First, the design field, $\mu$, is smoothed (giving $\hat{\mu}$ ) and projected (giving $\varphi=\widehat{\hat{\mu}}$ ). This projection defines the (polymer) base structure. In order to identify the interface of the base structure, a second smoothing is applied (giving $\hat{\varphi}$ ). A large norm of the spatial gradient, $\|\nabla \hat{\varphi}\|_{\alpha}$, in this second smoothed field defines the interface (the index $\alpha$ means that the norm is normalized, see section 2.1.2). The normalized norm is subsequently projected to model a sharp interface. This field, denoted $\overline{\|\nabla \hat{\varphi}\|_{\alpha}}$, defines the coating. Using this approach the desired coating thickness is defined indirectly by setting the filter radius used for the second smoothing, as this parameter determines the width of the interface region.

In the following subsections the above considerations will be developed in more detail.

\subsubsection{Filters}

Filters are a commonly used regularization technique in SIMP-based topology optimization to avoid mesh-dependency of solutions. In standard filtering 
techniques the filter radius, $R$, is equal to the radius of the neighborhood defined around each individual element for a filtering step. Certain filters assure a minimum feature size, e.g. the Heaviside projection (Guest et al. (2004)) for which the minimum feature size equals $2 R$. More details on standard filtering techniques may be found in Sigmund (2007).

In this work, where material interfaces are described by means of spatial gradients, the role of the filters is extended to provide the necessary control over the spatial gradient field. A density filter / smoothing and a projection scheme are applied.

\section{Smoothing}

The applied density filter is a so-called PDE-filter based on a Helmholtz-type partial differential equation (Lazarov and Sigmund (2011)). For the isotropic case the filtered density field, $\hat{\mu}$, is implicitly defined as a solution to the Helmholtz PDE

$$
-r^{2} \nabla^{2} \hat{\mu}+\hat{\mu}=\mu
$$

with appropriate boundary conditions. The scalar $r$ is a length scale parameter. The relation between $r$ and the filter radius, $R$, in standard filtering techniques is

$$
r=\frac{R}{2 \sqrt{3}}
$$

In this work the filter radius will be reported in terms of $R$ due to its more intuitive nature.

As opposed to standard filtering techniques which are solved for element density variables, the PDE-filter is solved for nodal density variables. The PDE filter has several advantages justifying the additional mapping needed between elemental and nodal densities: First, using nodal densities allows for an easy calculation of the spatial gradient and a convenient definition of boundary conditions. Second, the PDE-filter is suitable for parallel implementation which makes it a more attractive choice for large problems.

\section{Projection}

Projection methods (Guest et al. (2004), Sigmund (2007)) provide an efficient means of obtaining black-and-white designs. Wang et al. (2011) propose the following smoothed projection:

$$
\overline{\hat{\mu}}_{i}=\frac{\tanh (\beta \eta)+\tanh \left(\beta\left(\hat{\mu}_{i}-\eta\right)\right)}{\tanh (\beta \eta)+\tanh (\beta(1-\eta))}
$$


Here, $\beta$ determines the "sharpness" of the projection. $\eta \in[0 ; 1]$ is the threshold parameter. For $\eta=0$ and $\eta=1$ the projection reduces to the Heaviside step filter (Guest et al. (2004)) and modified Heaviside filter (Sigmund (2007)), respectively. In the limit of $\beta \rightarrow \infty$ these two threshold values provide length scale control for the solid and void phase, respectively. For intermediate values of $\eta$ there is no length scale control.

\section{Separating length scales by two-step filtering}

At sharp edges in the design field, the spatial density function is non-smooth and the gradient is not (analytically) defined. Using the gradient of the smoothed field eliminates this problem. When the PDE filter is applied (as in this work) the nodal densities are readily available for calculation of spatial gradients.

The two-step filtering process illustrated in Fig. 2 allows to separate the length scales of the base structure and the coating. First, the base structure is defined by a smoothing and subsequent projection of the design field. The smoothing is defined by the radius $R_{1}$, and the projection is controlled by the parameters $\beta$ and $\eta$. This filtering process provides an indirect length scale control of the base structure. In order to identify the interface of the base structure (represented by the projected field, $\varphi$ ), a second density filter is applied. By choosing the filter radius, $R_{2}$, of this second filter independently of $R_{1}$, the thickness of the coating may be decoupled and controlled independently of the feature size of the base structure. In order to model a sharp material interface, the normalized gradient norm of this second density filtered field, $\|\nabla \hat{\varphi}\|_{\alpha}$, is subsequently projected using the parameters $\beta_{g}$ and $\eta_{g}$ which may also be chosen independently of the parameters $\beta$ and $\eta$ from the first projection.

\subsubsection{Gradient norm}

The two-step filtering and interpolation approach illustrated in Fig. 2 involves the gradient norm for enforcing coating material at all interfaces between base material and void. More precisely, this gradient norm refers to the normalized Euclidean norm of the spatial gradient of the second smoothed field, $\hat{\varphi}$. The index $\alpha$ refers to the normalization, i.e.:

$$
\|\nabla \hat{\varphi}\|_{\alpha} \equiv \alpha\|\nabla \hat{\varphi}\|
$$

where the normalization factor $\alpha$ is defined as the inverse of the maximum possible gradient norm of the second smoothed field, $\hat{\varphi}$. This implies that

$$
0 \leq\|\nabla \hat{\varphi}\|_{\alpha} \leq 1, \quad \forall \varphi
$$


Derivation of the value of $\alpha$ which can be determined a priori is presented in section 2.1.4. It is the projection of this normalized gradient norm, $\|\nabla \hat{\varphi}\|_{\alpha}$, that is used for enforcing coating material.

\subsubsection{Interpolation functions}

Based on the above considerations, interpolation functions for the physical density and stiffness may be defined. The material properties of the base material are defined as a ratio of the coating material's properties:

1. Coating material: Mass density $m^{0}$, stiffness $E^{0}$

2. Base material: Mass density $\lambda_{m} m^{0}$, stiffness $\lambda_{E} E^{0}$

The coating material is assumed to have a higher stiffness and mass density than the base material, i.e. both $\lambda_{m}$ and $\lambda_{E}$ are contained in the interval $[0,1]$. The assumption is generally valid when considering polymer based structures with a metal coating. To make the connection with the standard SIMP interpolation as evident as possible, the mass density of the coating material is assumed to be $m^{0}=$ 1. Thereby the physical density and mass density may be used interchangeably, as the coating material has a mass density of 1 , whereas the base material has a mass density of $\lambda_{m}$. Both materials are assumed to be isotropic with a Poisson's ratio, $v^{0}$, independent of interpolation density.

The physical density, $\rho$, and stiffness, $E$, are defined as an interpolation of $\varphi$ and $\overline{\|\nabla \hat{\varphi}\|_{\alpha}}$ :

$$
\begin{gathered}
\rho\left(\varphi, \overline{\|\nabla \hat{\varphi}\|_{\alpha}}\right)=\lambda_{m} \varphi+\left(1-\lambda_{m} \varphi\right) \overline{\|\nabla \hat{\varphi}\|_{\alpha}} \\
E\left(\varphi, \overline{\|\nabla \hat{\varphi}\|_{\alpha}}\right)=E^{0}\left[\lambda_{E} \varphi^{p}+\left(1-\lambda_{E} \varphi^{p}\right)\left(\overline{\|\nabla \hat{\varphi}\|_{\alpha}}\right)^{p}\right]
\end{gathered}
$$

The penalization parameter, $p=3$, is the same for $\varphi$ and $\overline{\|\nabla \hat{\varphi}\|_{\alpha}}$. Note that when the gradient norm approaches zero, i.e. when going away from the interface, the expressions reduce to:

$$
\begin{aligned}
& \rho_{\text {base }}(\varphi, 0)=\lambda_{m} \varphi \\
& E_{\text {base }}(\varphi, 0)=E^{0} \lambda_{E} \varphi^{p}
\end{aligned}
$$

The similarity with standard SIMP is evident. At the other extreme where the normalized gradient norm approaches 1, i.e. at the interface region, the physical density and stiffness approach 1 and $E^{0}$, respectively:

$$
\begin{aligned}
& \rho_{\text {coating }}(\varphi, 1)=\lambda_{m} \varphi+\left(1-\lambda_{m} \varphi\right)=1 \\
& E_{\text {coating }}(\varphi, 1)=E^{0}\left[\lambda_{E} \varphi^{p}+\left(1-\lambda_{E} \varphi^{p}\right)\right]=E^{0}
\end{aligned}
$$


The physical density and stiffness functions are graphically represented in Fig. 3 and 4 for an example 1D design variable step function, $\mu$. There is a symmetry condition at the right edge, meaning that the figure represents (half of) a sharp edged feature. For the example, $\lambda_{E}<\lambda_{m}$. Remark that the slope at the interpolated edge is steeper for the stiffness function than for the physical density interpolation. This is due to the penalization of intermediate design densities as known from standard SIMP.

In addition to the properties given by Eq. (8) and (9) the following properties may be verified by insertion into (6) and (7) or by considering Fig. 3 and 4 :

$$
\begin{aligned}
& \rho(0,0)=0 \\
& \rho(1,0)=\lambda_{m}
\end{aligned}
$$

and

$$
\begin{aligned}
& E(0,0)=0 \\
& E(1,0)=\lambda_{E} E^{0}
\end{aligned}
$$

This follows the intention that base material is enforced where the design field equals 1 and has a low gradient. Equation (9) assures that coating material is enforced where the spatial gradient is large.

Note that unlike the standard SIMP interpolation, the stiffness is not defined as an explicit function of the physical density, $\rho$ (no bijective mapping exists between the physical density and stiffness). Instead, both the physical density and stiffness are interpolated based on the projected design variable field, $\varphi$, and the normalized gradient of the filtered field, $\overline{\|\nabla \hat{\varphi}\|_{\alpha}}$.

\subsubsection{Controlling gradients to achieve prescribed coating thickness}

Coating material is imposed at interfaces based on the normalized gradient norm of the second filtered field, $\hat{\varphi}$. This makes the modeled coating thickness, $t$, dependent on several variables.

The starting point is a given feature of width $w$ in the first projected field, $\varphi=\overline{\hat{\mu}}$. Note that $w$ varies throughout the field depending on which feature is considered. The field, and therefore $w$, depends on the filter radius, $R_{1}$, and the projection parameters, $\eta$ and $\beta$. The normalized gradient norm at the projected edge depends on the second filter radius, $R_{2}$, and the normalization factor, $\alpha$. Finally, the modeled coating is obtained by projecting the normalized gradient norm, making it also dependent on the second projection parameters, $\eta_{g}$ and $\beta_{g}$. In this section it is shown how the thickness, $t$, of the modeled coating can be 


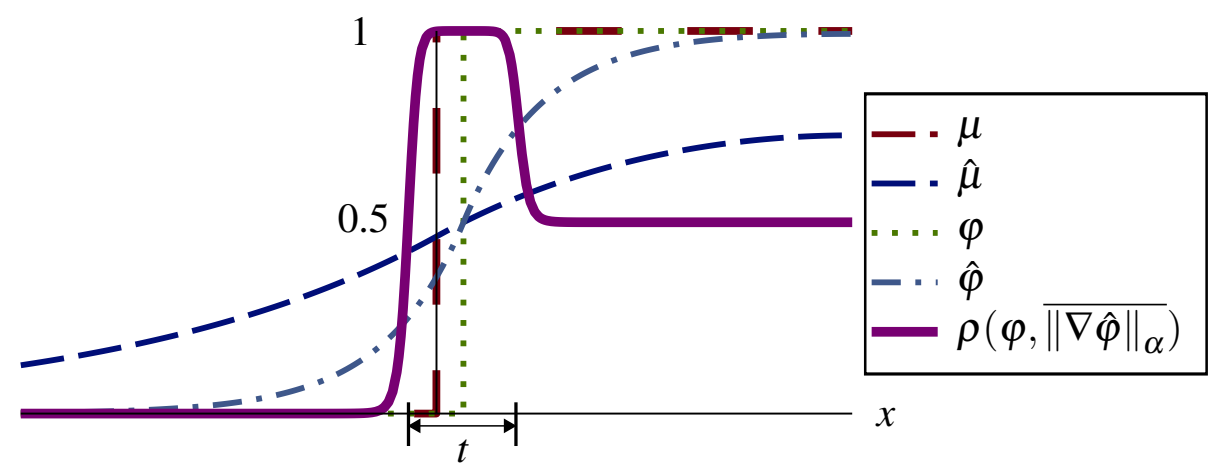

Figure 3: Physical density interpolation with underlying two-step filtered fields.

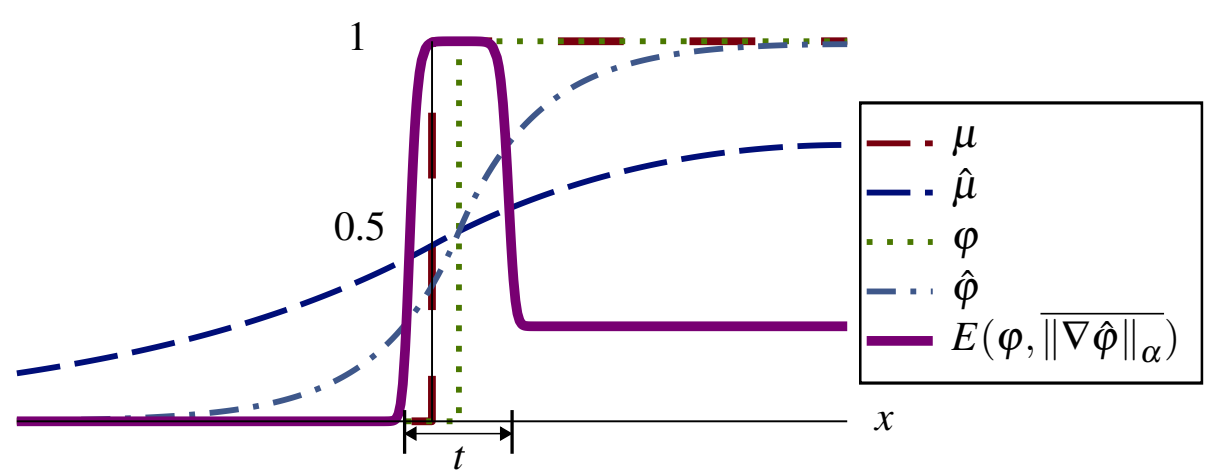

Figure 4: Physical stiffness interpolation with underlying two-step filtered fields. 
controlled to be equal to the prescribed coating thickness, $t_{r e f}$, through the second filter radius, $R_{2}$.

A first step is to derive an expression for the normalization factor, $\alpha$. This factor should be chosen as the inverse of the maximum possible value of the gradient norm in the second filtered field. Note that this value is a constant which is calculated before the optimization loop. To find this scaling value a simplified problem is considered in the following. The maximum will appear where the first projected field, $\varphi$, has a discrete (step) edge. Here the edge is assumed perpendicular to the $x$-direction. The edge should be straight and the feature should be of infinite extent in the $x$-direction. The extent in the $y$-direction may be of arbitrary, positive length, defined by the real valued limits $y_{1}$ and $y_{2}>y_{1}$. Such an edge may be represented as a Heaviside step function (see Fig. 5) in the limit of $\beta \rightarrow \infty$ :

$$
\varphi_{H}(x, y)=\mathrm{H}(x)
$$

where

$$
H(x)=\operatorname{Heaviside}(x)= \begin{cases}0 & x<0 \\ 1 & x>0\end{cases}
$$

The function is not defined for $x=0$.

Solving the PDE (1) with four homogeneous Neumann boundary conditions (at $y=y_{1}, y=y_{2}$ and the limits of $x \rightarrow \pm \infty$ ) gives the second filtered field

$$
\hat{\varphi}_{H}(x, y)=\mathrm{H}(x)+\frac{e^{x \frac{2 \sqrt{3}}{R_{2}}}}{2}(1-\mathrm{H}(x))-\frac{e^{-x \frac{2 \sqrt{3}}{R_{2}}}}{2} \mathrm{H}(x)
$$

This solution is independent of $y$ and identical to the solution when using the corresponding 1D step function

$$
\varphi_{H}(x)=\mathrm{H}(x)
$$

This implies that using the PDE filter allows to reduce length scale studies of the $2 \mathrm{D}$ problem to a $1 \mathrm{D}$ problem when the assumptions mentioned in this section are applied. This would not have been the case if a standard linear filter had been used.

The maximum gradient norm is obtained in the limit of $x \rightarrow 0$ as:

$$
\left\|\nabla \hat{\varphi}_{H}\right\|_{\max }=\frac{\sqrt{3}}{R_{2}}
$$




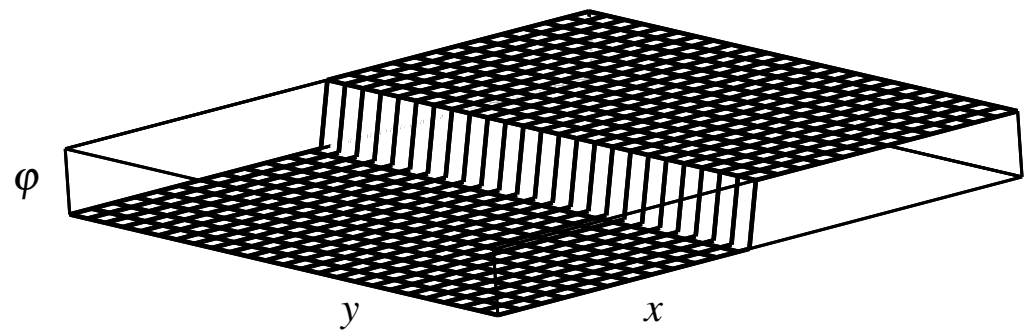

Figure 5: 2D step function representing the limit feature edge of infinite extent for calculation of maximum gradient.

giving a gradient normalization factor of

$$
\alpha=\frac{R_{2}}{\sqrt{3}}
$$

Based on this definition of $\alpha$ a relation between the second filter radius, $R_{2}$, and the modeled coating thickness, $t$, may the derived. The projection thresholds are chosen as $\eta_{g}=\eta=0.5$ and it is assumed that $\beta$ and $\beta_{g}$ are arbitrarily high. This implies that all projected fields have sharp edges. With these assumptions, the point limiting the coating from the base material or void is where $\|\nabla \hat{\varphi}\|_{\alpha}=\eta_{g}$. Based on this, the modeled coating thickness may be expressed as an explicit function of the filter radii, $R_{1}$ and $R_{2}$, and the width, $w$, of a sharp-edged feature in the design field. The analytical expression is rather lengthy and is omitted here, however, the analytical curves showing the thickness, $t$, as a function of $w$ are plotted for various values of $R_{1}$ and $R_{2}$ in Fig. 6. The modeled thickness converges quickly to a constant, maximum value, $t_{\max }$, which only depends on the second filter radius, $R_{2}$ :

$$
t_{\text {max }}=\frac{\ln (2)}{\sqrt{3}} R_{2}
$$

By exchanging this asymptotic value, $t_{\max }$, by the prescribed coating thickness, $t_{r e f}$, the required value of $R_{2}$ is obtained:

$$
R_{2}=\frac{\sqrt{3}}{\ln (2)} t_{r e f} \approx 2.5 t_{r e f}
$$

The larger the first filter radius, compared to the second, the narrower the transition band of $w$-values leading to intermediate coating thickness, i.e. between the minimum width, $w_{\min }$, and the width where the thickness reaches (a given percentage of) the prescribed coating thickness $t_{\max }=t_{\text {ref }}$. 


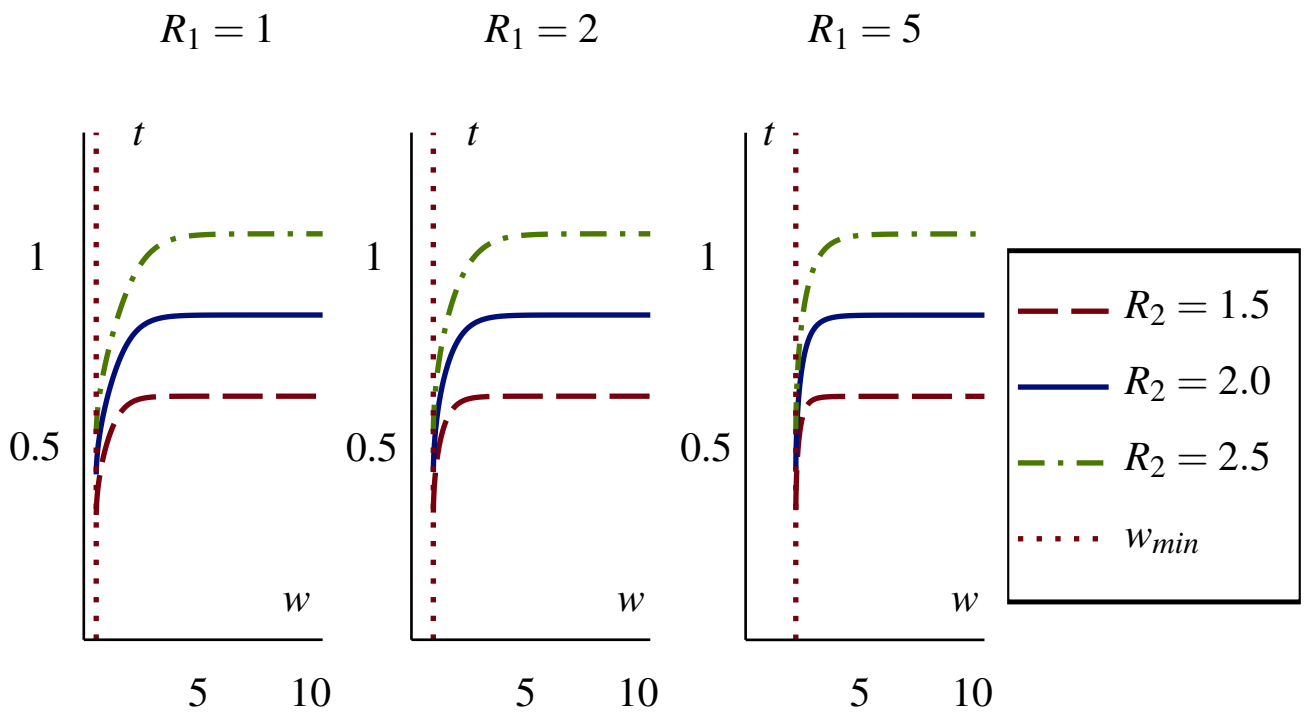

Figure 6: Modeled coating thickness, $t$, as a function of feature width, $w$, for a sharp-edged feature in the design field, $\mu$.

Sharp-edged features in the design field, $\mu$, with $w<w_{\min }$ will be filtered out by the first filtering and projection, meaning that the coating thickness is not defined for these values. Note that with the assumption of arbitrarily large $\beta$, features in the design field with smooth edges will be projected to have sharp edges in $\varphi$, and the features may be attributed an equivalent sharp-edged width.

\subsection{Optimization problem}

The optimization problem is a standard minimum compliance problem with a constraint on the volume. For the discretized problem the continuous fields (e.g. $\mu)$ are replaced with vectors of element values $(\boldsymbol{\mu})$.

The global stiffness matrix, $\mathbf{K}$, is defined as:

$$
\mathbf{K}(\boldsymbol{\mu})=\sum_{e} \mathbf{k}_{e}(\boldsymbol{\mu})=\sum_{e} E_{e}\left(\varphi_{e}(\boldsymbol{\mu}), \overline{\left\|\nabla \hat{\varphi}_{e}(\boldsymbol{\mu})\right\|_{\alpha}}\right) \mathbf{k}_{e}^{0}
$$

where $\mathbf{k}_{e}$ is the element stiffness matrix, and $\mathbf{k}_{e}^{0}$ is the element stiffness matrix for an element with unit Young's modulus. For the numerical implementation a small minimum stiffness $\left(\lambda_{E, \min } E^{0}\right)$ in void elements is required to avoid singularity. The first term in the stiffness interpolation, $\lambda_{E} \varphi_{e}^{p}$, is replaced with $\lambda_{E, \min }+\left(\lambda_{E}-\right.$ $\left.\lambda_{E, \min }\right) \varphi_{e}^{p}$. 
The optimization problem is defined in the following way:

$$
\begin{aligned}
\min _{\boldsymbol{\mu}}: & c(\boldsymbol{\mu})=\mathbf{U}^{\mathbf{T}} \mathbf{K U} \\
\text { subject to: } & \mathbf{K U}=\mathbf{F} \\
& g(\boldsymbol{\mu})=V(\boldsymbol{\mu}) / V^{*}-1 \leq 0 \\
& 0 \leq \mu_{e} \leq 1, \quad \forall e
\end{aligned}
$$

Here $c$ is the compliance, $\mathbf{U}$ and $\mathbf{F}$ are the global displacement and force vectors, respectively, $g$ is the volume constraint, $V(\boldsymbol{\rho}(\boldsymbol{\mu}))=\sum v_{i} \rho_{i}(\boldsymbol{\mu})$ is the material volume and $V^{*}$ is the maximum allowed volume.

Design updates are performed based on sensitivities using MMA (Svanberg (1987)).

\subsection{Sensitivity analysis}

For the sensitivity analysis the explicit statement of variable dependency is omitted, i.e. the long notation $\rho\left(\varphi, \overline{\|\nabla \hat{\varphi}\|_{\alpha}}\right)$ used in previous sections is replaced with the shorter $\rho$ etc. Furthermore, the following notation is introduced:

$$
E=E^{0}\left[\varepsilon^{S}+\left(1-\varepsilon^{S}\right) \varepsilon^{G}\right]=E^{0}\left[\varepsilon^{S}+\varepsilon^{G}-\varepsilon^{S} \varepsilon^{G}\right]
$$

where $S$ refers to "SIMP", and $G$ refers to "gradient":

$$
\varepsilon^{S}=\lambda_{E} \varphi^{p}, \quad \varepsilon^{G}=\left(\overline{\|\nabla \hat{\varphi}\|_{\alpha}}\right)^{p}
$$

Using the adjoint method, the sensitivity of the objective function with respect to an element design variable is:

$$
\frac{\partial c}{\partial \mu_{e}}=-\mathbf{U}^{T} \frac{\partial \mathbf{K}}{\partial \mu_{e}} \mathbf{U}=\sum_{i} \frac{\partial E_{i}}{\partial \mu_{e}}\left(-\mathbf{u}_{i}^{T} \mathbf{k}^{0} \mathbf{u}_{i}\right)
$$

with

$$
\frac{\partial E_{i}}{\partial \mu_{e}}=E^{0}\left[\frac{\partial \varepsilon_{i}^{S}}{\partial \mu_{e}}+\frac{\partial \varepsilon_{i}^{G}}{\partial \mu_{e}}-\frac{\partial\left(\varepsilon_{i}^{S} \varepsilon_{i}^{G}\right)}{\partial \mu_{e}}\right]
$$

The first derivative term is obtained using the chain rule:

$$
\frac{\partial \varepsilon_{i}^{S}}{\partial \mu_{e}}=\frac{\partial \varepsilon_{i}^{S}}{\partial \varphi_{i}} \frac{\partial \varphi_{i}}{\partial \mu_{e}}=p \varphi_{i}^{p-1} \lambda_{E} \frac{\partial \varphi_{i}}{\partial \mu_{e}}
$$


Note that when using the minimum stiffness $\lambda_{E, \min }$, the factor $\lambda_{E}$ should be modified to $\left(\lambda_{E}-\lambda_{E, \text { min }}\right)$ in (26). The term $\partial \varphi_{i} / \partial \mu_{e}$ represents the standard modification of sensitivities due to filtering and projection. The expression is omitted here but may be found in the respective papers (Lazarov and Sigmund (2011) for the PDE-filter and Wang et al. (2011) for the projection).

The second derivative term of (25) is elaborated in a similar way:

$$
\frac{\partial \varepsilon_{i}^{G}}{\partial \mu_{e}}=\frac{\partial \varepsilon_{i}^{G}}{\partial\left(\overline{\left\|\nabla \hat{\varphi}_{i}\right\|_{\alpha}}\right)} \frac{\partial\left(\overline{\left\|\nabla \hat{\varphi}_{i}\right\|_{\alpha}}\right)}{\partial \mu_{e}}=p\left(\overline{\left\|\nabla \hat{\varphi}_{i}\right\|_{\alpha}}\right)^{p-1} \frac{\partial\left(\overline{\left\|\nabla \hat{\varphi}_{i}\right\|_{\alpha}}\right)}{\partial \mu_{e}}
$$

The last derivative term of $(27)$ is further developed using the chain rule:

$$
\frac{\partial\left(\overline{\left\|\nabla \hat{\varphi}_{i}\right\|_{\alpha}}\right)}{\partial \mu_{e}}=\frac{\partial\left(\overline{\left\|\nabla \hat{\varphi}_{i}\right\|_{\alpha}}\right)}{\partial\left(\left\|\nabla \hat{\varphi}_{i}\right\|_{\alpha}\right)} \frac{\partial\left(\left\|\nabla \hat{\varphi}_{i}\right\|_{\alpha}\right)}{\partial \mu_{e}}
$$

Again, the factor $\partial\left(\overline{\left\|\nabla \hat{\varphi}_{i}\right\|_{\alpha}}\right) / \partial\left(\left\|\nabla \hat{\varphi}_{i}\right\|_{\alpha}\right)$ is a standard filter modification factor. The derivative of the normalized gradient norm (the second factor in (28)) is:

$$
\frac{\partial\left(\left\|\nabla \hat{\varphi}_{i}\right\|_{\alpha}\right)}{\partial \mu_{e}}=\frac{\alpha}{\left\|\nabla \hat{\varphi}_{i}\right\|}\left(\frac{\partial \hat{\varphi}_{i}}{\partial x} \frac{\partial}{\partial \mu_{e}}\left(\frac{\partial \hat{\varphi}_{i}}{\partial x}\right)+\frac{\partial \hat{\varphi}_{i}}{\partial y} \frac{\partial}{\partial \mu_{e}}\left(\frac{\partial \hat{\varphi}_{i}}{\partial y}\right)\right)
$$

This expression involves the two gradient components of the second smoothed field:

$$
\nabla \hat{\varphi}_{i}=\left(\begin{array}{c}
\frac{\partial \hat{\varphi}_{i}}{\partial x} \\
\frac{\partial \hat{\varphi}_{i}}{\partial y}
\end{array}\right)=\left(\begin{array}{c}
\frac{\partial}{\partial x} \\
\frac{\partial}{\partial y}
\end{array}\right) \mathbf{N}^{\mathbf{T}} \hat{\boldsymbol{\xi}}_{\boldsymbol{i}}=\mathbf{B} \hat{\boldsymbol{\xi}}_{\boldsymbol{i}}
$$

Here, $\hat{\boldsymbol{\xi}}_{i}$ is a vector of the four nodal densities of the second smoothed field corresponding to element $i$. Note that the nodal densities are obtained directly when using the PDE-filter (see section 2.1.1). $\mathbf{N}$ is a vector of the four shape functions relating $\hat{\boldsymbol{\xi}}_{i}$ with $\hat{\varphi}_{i}$, and $\mathbf{B}^{2 x 4}$ is the gradient computation matrix for which the two rows are obtained by differentiating the four shape functions with respect to the two spatial variables, $x$ and $y$, respectively.

Eq. (29) also involves the derivative with respect to the design variables of the partial, spatial derivatives. As the terms in $x$ and $y$ are identical only the term in $x$ is developed further here. Based on the gradient calculation shown in Eq. (30) and denoting the two rows of $\mathbf{B}$ by $\mathbf{B}^{x}$ and $\mathbf{B}^{y}$, respectively, the following expression is obtained:

$$
\frac{\partial}{\partial \mu_{e}}\left(\frac{\partial \hat{\varphi}_{i}}{\partial x}\right)=\frac{\partial}{\partial \mu_{e}}\left(\mathbf{B}^{x} \hat{\boldsymbol{\xi}}_{\boldsymbol{i}}\right)=\sum_{k=1}^{4} \mathbf{B}_{k}^{x} \frac{\partial \hat{\xi}_{i k}}{\partial \mu_{e}}
$$


The factor $\partial \hat{\xi}_{i k} / \partial \mu_{e}$ is a standard filter modification factor. The calculation of this term is described in detail in Lazarov and Sigmund (2011).

The last derivative term of $(25)$ is obtained simply by using the product rule:

$$
\frac{\partial\left(\varepsilon_{i}^{S} \varepsilon_{i}^{G}\right)}{\partial \mu_{e}}=\frac{\partial \varepsilon_{i}^{S}}{\partial \mu_{e}} \varepsilon_{i}^{G}+\varepsilon_{i}^{S} \frac{\partial \varepsilon_{i}^{G}}{\partial \mu_{e}}
$$

Expressions for all terms have already been given above.

\section{Results}

In the following a range of numerical results are presented. Many parameters are identical for all examples: The penalization $p=3$ is used for both $\varphi$ and $\overline{\|\nabla \hat{\varphi}\|_{\alpha}}$. The two projections are performed with identical parameters. The threshold is $\eta=\eta_{g}=0.5$, and the sharpness parameter is initialized with $\beta=8$. This number should be sufficiently high to make the projected gradient have an impact already from the beginning of the optimization. A continuation scheme is adopted, where $\beta$ is gradually increased to 64 by doubling at every 100th iteration (or at convergence).

\subsection{MBB beam}

The first numerical example is the MBB benchmark example. The design domain is shown in Fig. 7). A few, minor modifications of the standard design domain are introduced: First, homogeneous Dirichlet boundary conditions have been prescribed for the PDE filter to force a gradient at outer boundaries. Symmetry constraints have homogeneous Neumann conditions. Second, solid material (coating material) is required at all loads and supports to overrule the zero Dirichlet condition. For a point load or support, the width of the solid box should be larger than the filter radius. This simultaneously removes the large, local deformations that would appear and should be modeled if a thin shell is subdued a perpendicular, concentrated load (same principle as a fiber reinforced sandwich structure). At solid boxes a Dirichlet boundary condition of $\hat{\mu}=1$ is prescribed. Identical boundary conditions are used for the two density filtering steps. The domain has the dimensions 150 by 50 and is discretized by square elements with varying refinement.

The problem is optimized using a volume fraction of $40 \%$, a filter radius $R_{1}=$ 10 and a coating thickness $t_{r e f}=2$, i.e. $R_{2}=5$. The material parameters are $E^{0}=$ $1, \lambda_{m}=0.7$ and $\lambda_{E}=0.4$, meaning that the coating material is disproportionally stiff with respect to its weight compared to the base material. 


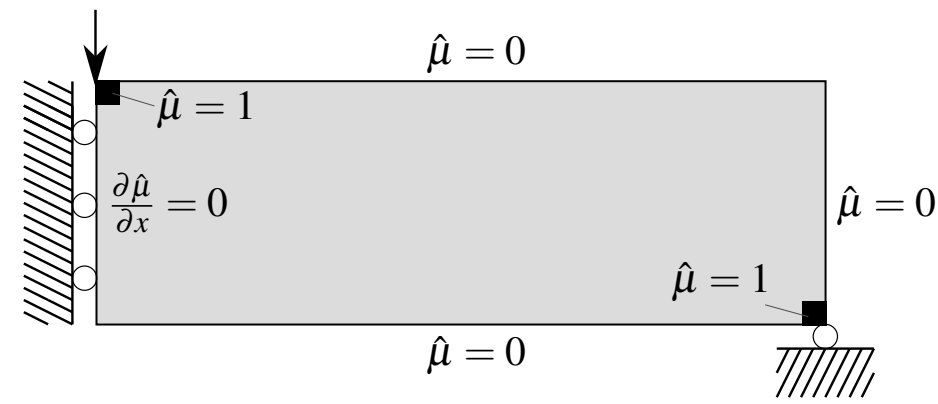

Figure 7: Design domain for MBB beam, including (nodal) boundary conditions for PDE filter.

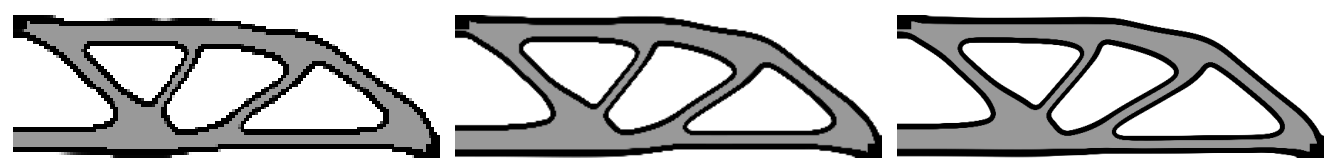

Figure 8: Optimized MBB beam. Left: 150 by 50 elements $(c=324.3)$. Middle: 300 by 100 elements $(c=305.2)$. Right: 600 by 200 elements $(c=305.8)$.

Fig. 8 shows the optimized structure using three different discretizations with element side lengths of $1,0.5$ and 0.25 , respectively. The three designs have the same topology and are very similar in shape. The coating thickness is highly uniform and almost independent of discretization.

Note that the figure shows the stiffness ( $E$-field) of the structures. For comparison, Fig. 9 shows the physical density field, $\rho$, of the middle structure. The base material has a relative stiffness value of $\lambda_{E}=0.4$ but a relative physical density value of $\lambda_{m}=0.7$.

A detail of the coating for the three designs is shown in Fig. 10. The coating thickness is seen to be identical for the three designs, only the resolution is improved. Within the precision of the resolution, and accounting for variations due to the FE discretization, the thicknesses equal the prescribed coating thickness, $t_{r e f}=2.0$. For an axis-parallel edge in the three designs shown in Fig. 8, this maximum value corresponds to 2, 4 and 8 elements, respectively.

If the resolution becomes even coarser, the diagonal edges would begin to con-

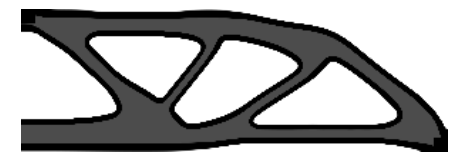

Figure 9: Physical density field, $\rho$, of optimized MBB beam using 300 by 100 elements. 

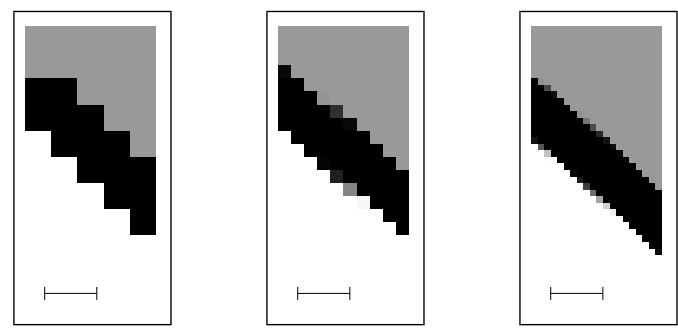

Figure 10: Detail of Fig. 8, showing leftmost member. Scale bar indicates $t_{r e f}=2.0$.
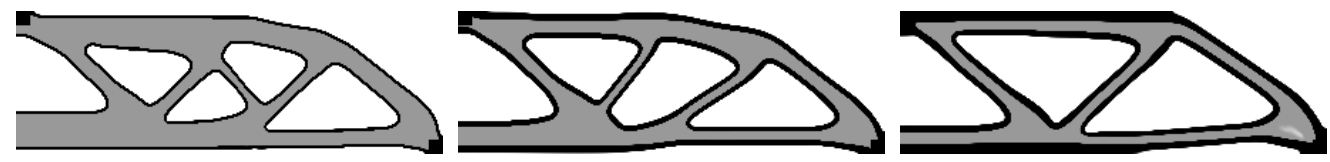

Figure 11: Optimized MBB beam with varying coating thickness. Left: $t_{\text {ref }}=1 \Rightarrow R_{2}=2.5$ $(c=374.4)$. Middle: $t_{\text {ref }}=2 \Rightarrow R_{2}=5(c=305.2)$. Right: $t_{\text {ref }}=3 \Rightarrow R_{2}=7.5(c=270.2)$.

tain one-node connections in the coating which would not make physical sense. In general it is recommended to resolve coating features with at least two elements in order for the model to maintain its physical meaning. Assuming a coating thickness everywhere of $t_{r e f}$, denoting the longer of the element side lengths as $w_{e}$, and using the relation (19), this implies a resolution requirement, i.e. element size, of

$$
2 w_{e} \leq t_{r e f} \Rightarrow w_{e} \leq \frac{t_{r e f}}{2}=\frac{\ln 2}{2 \sqrt{3}} R_{2}
$$

When choosing $R_{2}$ close to this limit, $R_{1}$ should be greater than or equal $R_{2}$ to assure sufficiently wide features in the base structure.

In Fig. 11 the same design problem as above is solved with the intermediate discretization ( 300 by 100 elements) and varying coating thickness. The modeled coating thickness is clearly controlled by modifying the filter radius, $R_{2}$. The compliance changes as a thicker coating takes up more material per unit length. The compliance improves significantly when allowing for a thicker coating, and thereby reducing the amount of base material which is disproportionately weak. The design is gradually approaching the design obtained with a standard SIMP optimization. Using the same method as above to calculate the coating thickness, the relation to Eq. (18) may be verified.

As indicated in the analytical analysis of the modeled coating thickness, illustrated in Fig. 6 from section 2.1.4, the modeled coating thickness at a given interface point depends on the feature size, $w$, of the underlying feature in the first 

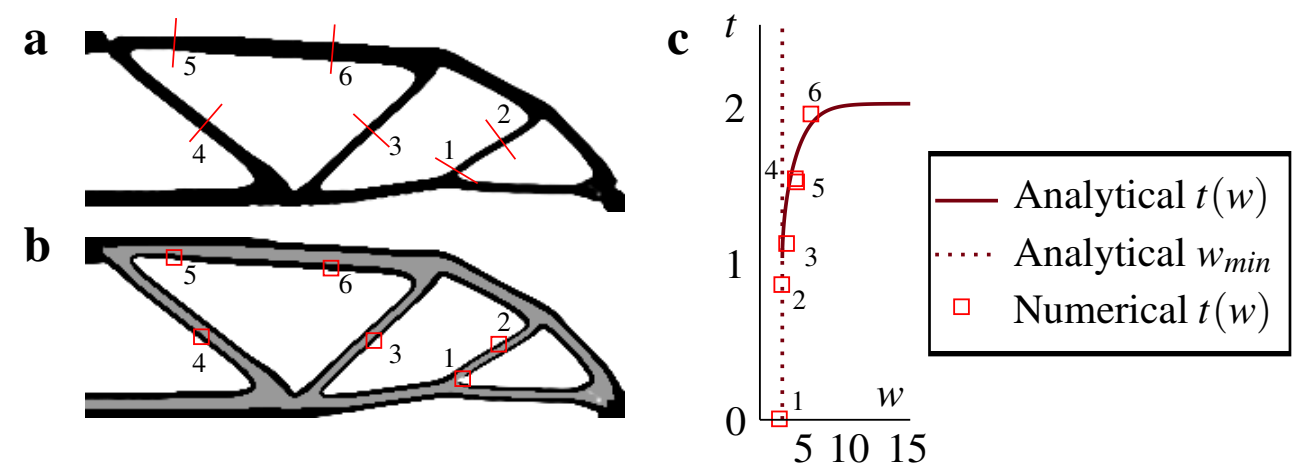

Figure 12: Dependence of modeled coating thickness, $t$, on the feature size, $w$ (measured in the first projected field, $\varphi$ ). a First projected field, $\varphi$. b Physical stiffness field, $E$. c Comparison of numerical values obtained in (a) and (b) vs. analytical curve derived in section 2.1.4.

projected field, $\varphi$. The figure illustrates that a certain feature size is needed in order to obtain a final design with constant modeled coating thickness. Thinner features are predicted to have a thinner modeled coating thickness. In Fig. 12 this circumstance has been investigated from a numerical perspective. The same problem as in Fig. 8 is considered, except that the volume fraction is decreased to $30 \%$ and the first filter radius is decreased to $R_{1}=5$. The problem is solved using the intermediate discretization. The optimized structure is shown in Fig. 8, For six cross-sections of varying width, the numerical values of $w$ and $t$ have been calculated. In the figure the values are compared to the analytically derived relation. The numerical results closely follow the analytical prediction to within the precision of the discretization. In order to be guaranteed a constant modeled coating thickness for the entire design a sufficiently high minimum feature size is needed. For most practical applications the coating thickness will be much smaller (at least several times smaller) than the base structure feature size. This implies a value of $R_{2}$ which is correspondingly low compared to $R_{1}$, meaning that the problem of non-constant modeled coating thickness is less likely to appear.

\subsection{Design dependency on material properties}

The topology of the optimized design is dependent on the values of $\lambda_{E}$ and $\lambda_{m}$. The effect of varying the relative physical density, $\lambda_{m}$, for a fixed relative stiffness, $\lambda_{E}=0.2$, is shown in Fig. 13 . The filter radii are $R_{1}=R_{2}=3.5$, corresponding to a maximum coating thickness of $t_{\max }=1.4$. The volume constraint is $40 \%$.

The figure illustrates an important point: When the value of $\lambda_{m}$ is chosen too close to the volume constraint for the problem, the entire design domain may be 


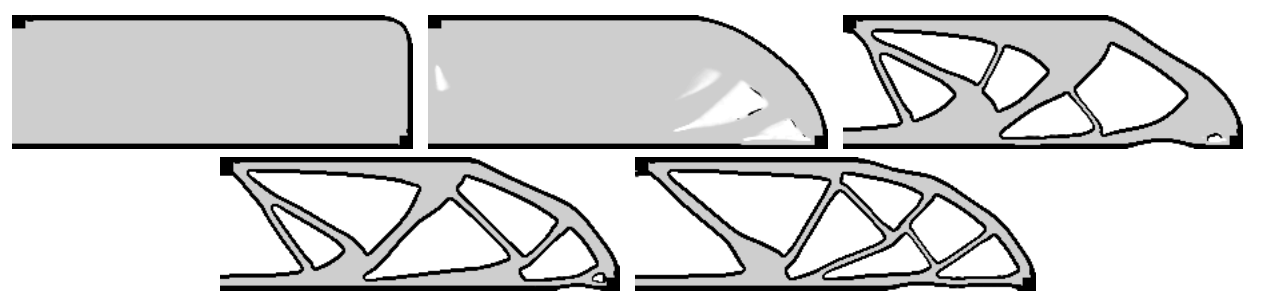

Figure 13: Effect of varying $\lambda_{m}$ with fixed $\lambda_{E}=0.2$. Values of $\lambda_{m}$ (from left to right): $0.2,0.4$, $0.6,0.8,0.99$.

filled up with base material while keeping below the maximum amount of volume allowed. This is what happens in the first structure of Fig. 13 with $\lambda_{m}=0.2$. The second structure with $\lambda_{m}=0.4$ shows the behavior around the limit value where the volume constraint becomes active. The coating is only partially created. This is possible because there is no minimum length scale control in the first projected design field, meaning that the interface may be blurred sufficiently to make the gradient norm attain a value below the projection threshold. It is a well-known issue in SIMP-based topology optimization that the volume constraint has to be active in order for the penalization to remove intermediate design densities. The issue may be alleviated using robust design schemes (e.g. Wang et al. (2011)) but this is outside the scope of this paper.

For larger values of $\lambda_{m}$ the optimized structures have a clearly defined and homogeneous coating. Base material becomes more and more uneconomical as $\lambda_{m}$ increases. This favors generation of a higher amount of coating material, implying an increased number of holes. However, simultaneously the total amount of base material available decreases in order for the volume constraint to be satisfied. This impedes the structure in branching out in a high number of members, thus limiting the number of holes and counteracting the other effect.

Fig. 14 shows compliance iso-curves obtained by varying the values of $\lambda_{m}$ and $\lambda_{E}$. The dashed line indicates the minimum value, $\lambda_{m}=0.33$, required to make the volume constraint active for this problem. Structures optimized with a value of $\lambda_{m}$ below this value will attain the same topology, independent of the choice of $\lambda_{E}$ (see example structures in the figure). However, the stiffness will still vary with $\lambda_{E}$. As a result, left of the dashed line all compliance iso-curves are horizontal.

For the part of the figure to the right of the dashed line, the iso-curves appear as almost straight lines with varying slope. The higher the value of $\lambda_{E}$ and the lower the value of $\lambda_{m}$, the lower the compliance. Furthermore, the lower the compliance value, the larger the distance between iso-lines as the relative change becomes 


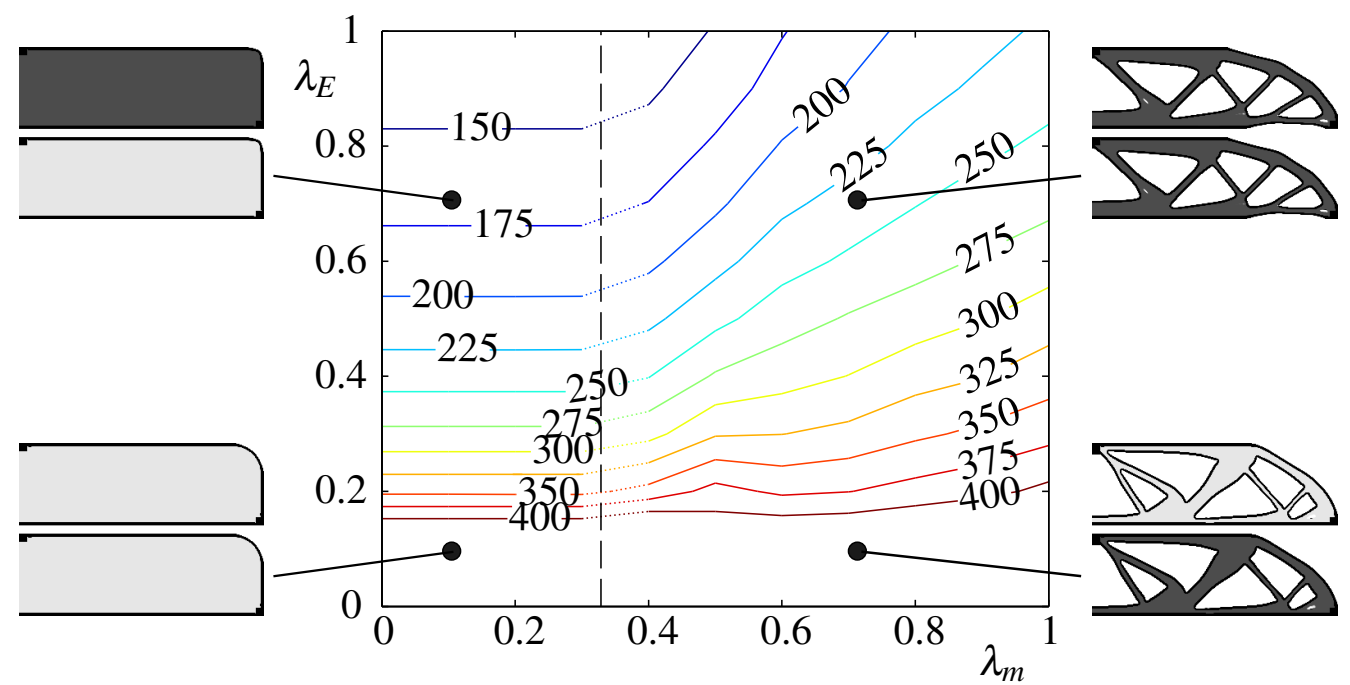

Figure 14: Compliance iso-curves when varying $\lambda_{m}$ and $\lambda_{E}$. For the four example structures the upper figure shows the stiffness and the lower shows the physical density. The dashed line indicates the minimum $\lambda_{m}$ required to make the volume constraint active.

larger.

\subsection{Bi-axial tension}

A second test example is the optimization of a square plate in bi-axial tension. The design domain is shown in Fig. 15. For symmetry reasons only one quarter of the domain is modeled. The shown domain has dimensions 100 by 100 and is discretized using 200 by 200 square elements. The distributed load adds up to 1 for a full plate side length. The problem is optimized for varying filter radius, $R_{1}$, and relative stiffness of base material, $\lambda_{E}$. The coating thickness is set to $t_{r e f}=1$ $\left(R_{2}=2.5\right)$, and the volume fraction is $30 \%$. The material parameters are $E^{0}=1$ and $\lambda_{m}=0.75$.

Fig. 16 shows the optimized structures. All designs are symmetrical and have a clearly defined coating. In the top row the relative stiffness, $\lambda_{E}$, of the base material is lower than in the bottom row. This makes coating material more attractive and the result is a higher amount of interface. In the right figures, the filter radius for the first smoothing is only half of the value used in the left figures, thereby allowing for smaller and finer features.

\subsection{Multiple load cases}

Fig. 17 shows a bridge-like design problem involving multiple load cases. The structure is simply supported and the five vertical unit loads are applied individu- 


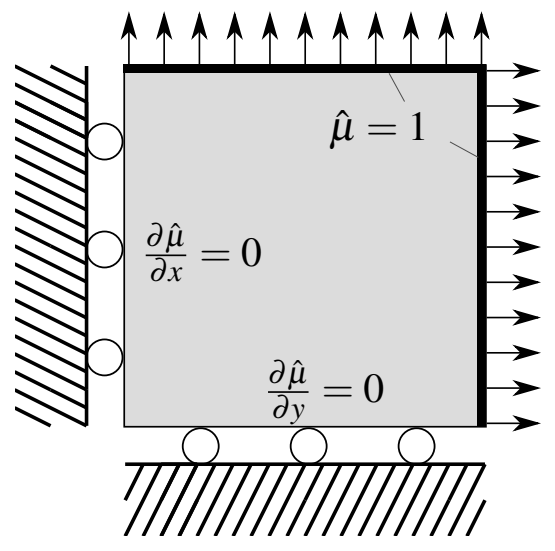

Figure 15: Design domain for plate in bi-axial tension, including (nodal) boundary conditions for PDE filter.

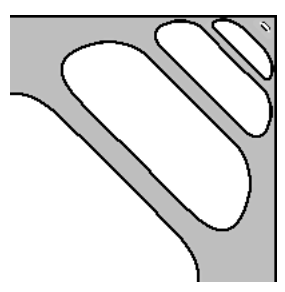

(a) $R_{1}=5, \lambda_{E}=0.25, c=5.18$

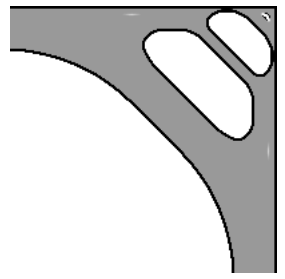

(c) $R_{1}=5, \lambda_{E}=0.40, c=4.20$

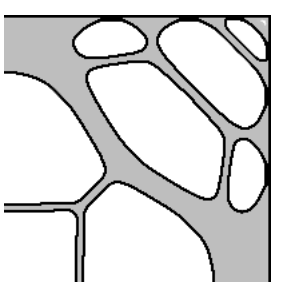

(b) $R_{1}=2.5, \lambda_{E}=0.25, c=4.82$

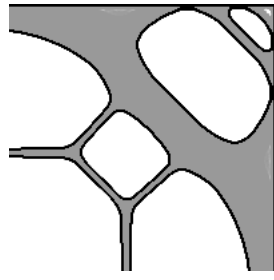

(d) $R_{1}=2.5, \lambda_{E}=0.40, c=4.01$

Figure 16: Optimized plate under bi-axial loading. 


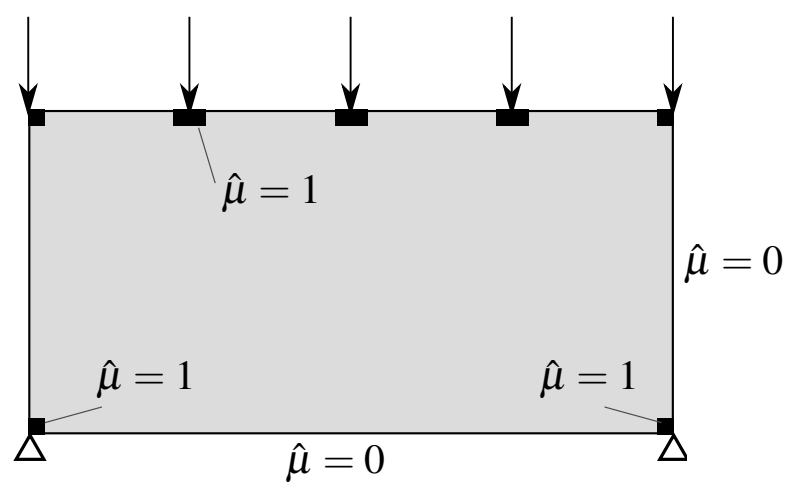

Figure 17: Design domain for problem with five vertical load cases, including (nodal) boundary conditions for PDE filter.

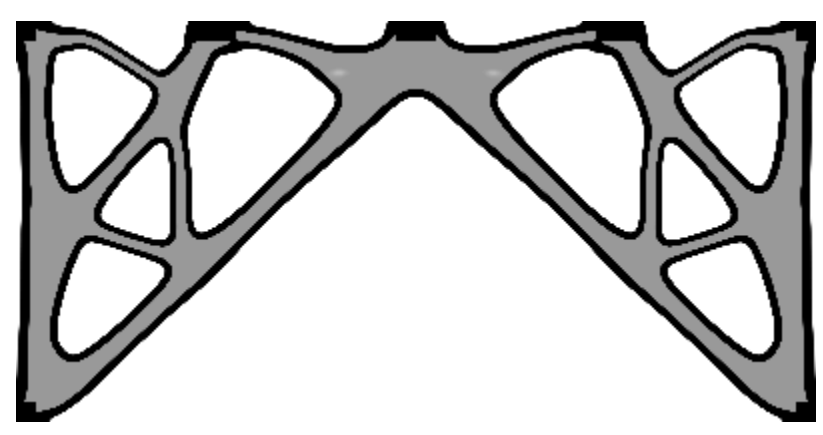

Figure 18: Optimized structure for 5 vertical load cases. Average compliance for the five load cases: $c=30.8$.

ally. The objective function is defined as the sum of the corresponding compliance values. The domain is rectangular with dimensions 200 by 100 and is discretized with two elements per unit length. The maximum amount of volume allowed is $30 \%$, the first filter radius is $R_{1}=10$ and the coating thickness is $t_{r e f}=2\left(R_{2}=5\right)$. The material parameters are $\lambda_{E}=0.4, \lambda_{m}=0.75$.

The optimized structure is shown in Fig. 18. As it would have been the case if a standard black and white projection method was used, the optimized structure consists of triangular holes only. This makes the design perform well for each of the individual load cases. Again, the coating is successfully applied uniformly at all interfaces.

\subsection{Microstructural infill using Hashin-Shtrikman bounds}

A particular application for the approach presented in this paper is the design of structures or components made of a single material, but where only the surface 
is required to be solid. The interior is instead required to have a fixed, pre-defined porosity. In additive manufacturing, this situation is frequently encountered: In the standard format of many 3D printers, a 3D object is defined by its surface. When preparing the print, the surface thickness and infill percentage are specified. These parameters may be directly adopted into the approach of this paper: The surface thickness translates into a coating thickness, $t_{r e f}$ (giving the second filter radius, $R_{2}$ ), and the infill percentage gives $\lambda_{m}$ and $\lambda_{E}$. This approach allows to cheaply include microstructure into the optimization model by using only the homogenized properties.

When the porous infill is required to be isotropic and made of the same material as the solid shell the relation between the material density, $\lambda_{m}$, and stiffness, $\lambda_{E}$, should satisfy the Hashin-Shtrikman (HS) bounds in order to be physically meaningful (Hashin and Shtrikman (1963)). In 2D, the HS bounds for the Young's modulus, $E$, are given by (Torquato et al. (1998)):

$$
0 \leq E \leq E^{*}(\rho)=\frac{\rho E_{0}}{3-2 \rho}
$$

where $\rho$ is the density and $E_{0}$ is the Young's modulus for full material density $(\rho=1)$. A porous material of density $\rho=\lambda_{m}$ which exactly satisfies the HS upper bound will thus have a normalized Young's modulus, $\lambda_{E}$, of

$$
\lambda_{E}=\frac{E^{*}\left(\lambda_{m}\right)}{E_{0}}=\frac{\lambda_{m}}{3-2 \lambda_{m}}
$$

Fig. 19 shows the HS upper bound normalized with respect to $E_{0}$ as a function of the material density. For single material applications, chosen combinations of $\left(\lambda_{m}, \lambda_{E}\right)$ should lie below the curve. Bendsøe and Sigmund (1999) realize microstructures consisting of solid material and void for a range of combinations of these parameters.

In Fig. $20 \mathrm{a}-\mathrm{b}$ the plate from section 3.3 is optimized using infill material with properties corresponding to the HS upper bound. The relative stiffness, $\lambda_{E}$, and the filter radius are shown in the figure. The relative density, $\lambda_{m}$, is determined from $\lambda_{E}$ based on (35). For $\lambda_{E}=0.25$ the corresponding relative density is $\lambda_{m}=$ 0.5 and for $\lambda_{E}=0.4$ the density is $\lambda_{m}=0.67$. All other parameters are the same as in section 3.3 . The obtained topologies resemble those obtained in section 3.3 .

In Fig. 20k-d the same problem is solved with a standard projection filter. With the formulation used in this paper it corresponds to setting the gradient normalization factor $\alpha$ to zero. The topologies are different than for the coated designs. The 


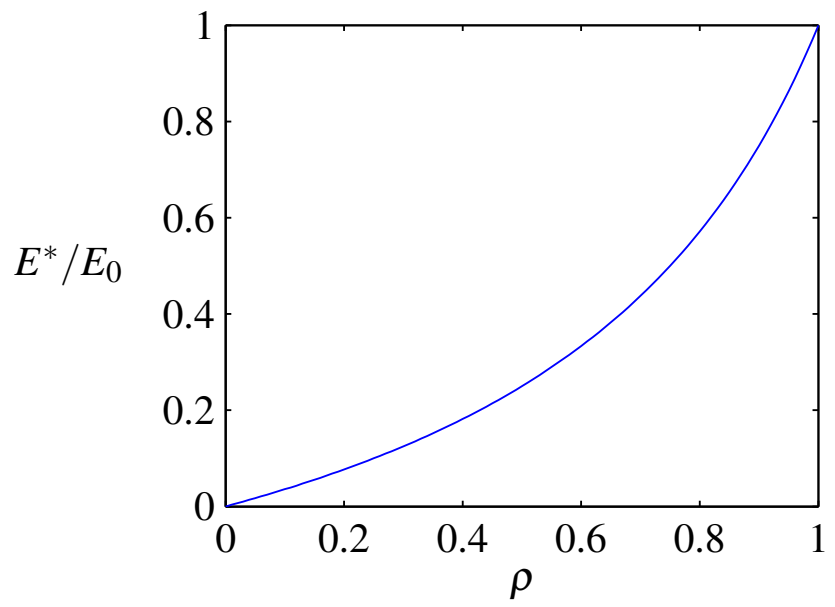

Figure 19: Hashin-Shtrikman upper bound of stiffness as a function of material density.

right structure contains some gray areas. The compliance values for these structures are both lower than the corresponding values for the solid surface structures optimized with the same filter radius, $R_{1}$ (by $19 \%$ and $12 \%$, respectively).

A second example is the T-domain shown in Fig. 21. The domain has the dimensions indicated in the figure. The problem is discretized with square elements of side length $1 / 8$. The first filter radius is $R_{1}=2$ and the coating thickness is set to $t_{r e f}=0.4$ giving a second filter radius of $R_{2}=1$. The volume fraction is set to $40 \%$. The horizontal load which is distributed at the bottom face sums up to 1. Again, the relative material parameters for the base material are set such that the HS upper bound is attained. In line with the plate example this problem is optimized both using $\lambda_{E}=0.4$ and with a standard projection optimization.

The optimized structures are shown in Fig. 22. The topologies for the two examples are rather similar, however, several of the holes from the standard projection solution have been closed when using the solid surface method ( $\left.\lambda_{E}=0.4\right)$. At the top center the holes have completely disappeared. At the top of the vertical member, the holes for the cross beams are only just initiated. Again, the compliance for the standard projection structure is lower $(8 \%)$ than for the solid surface structure.

A range of single material example cases have been run in relation to this paper. For all cases a pure black and white design performs better than a design with a solid surface and porous infill material attaining the HS upper bound. The reason is that the microstructure used for the infill is predefined and required to be isotropic. This corresponds to putting a strong constraint on the microstructure, as 


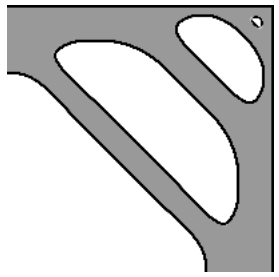

(a) $R_{1}=10, \lambda_{E}=0.40, c=3.60$

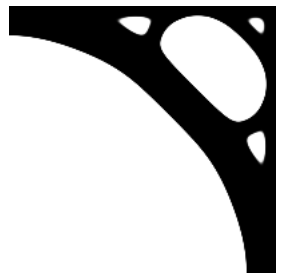

(c) $R_{1}=10$, std. proj., $c=2.92$

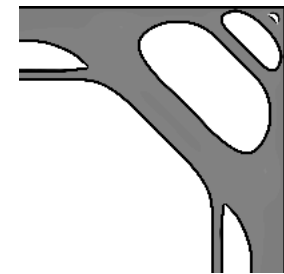

(b) $R_{1}=5, \lambda_{E}=0.50, c=3.51$

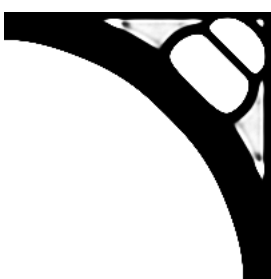

(d) $R_{1}=5$, std. proj., $c=3.10$

Figure 20: Optimized plate under bi-axial loading. a-b Using infill attaining the HS upper bound. c-d Using standard projection method.

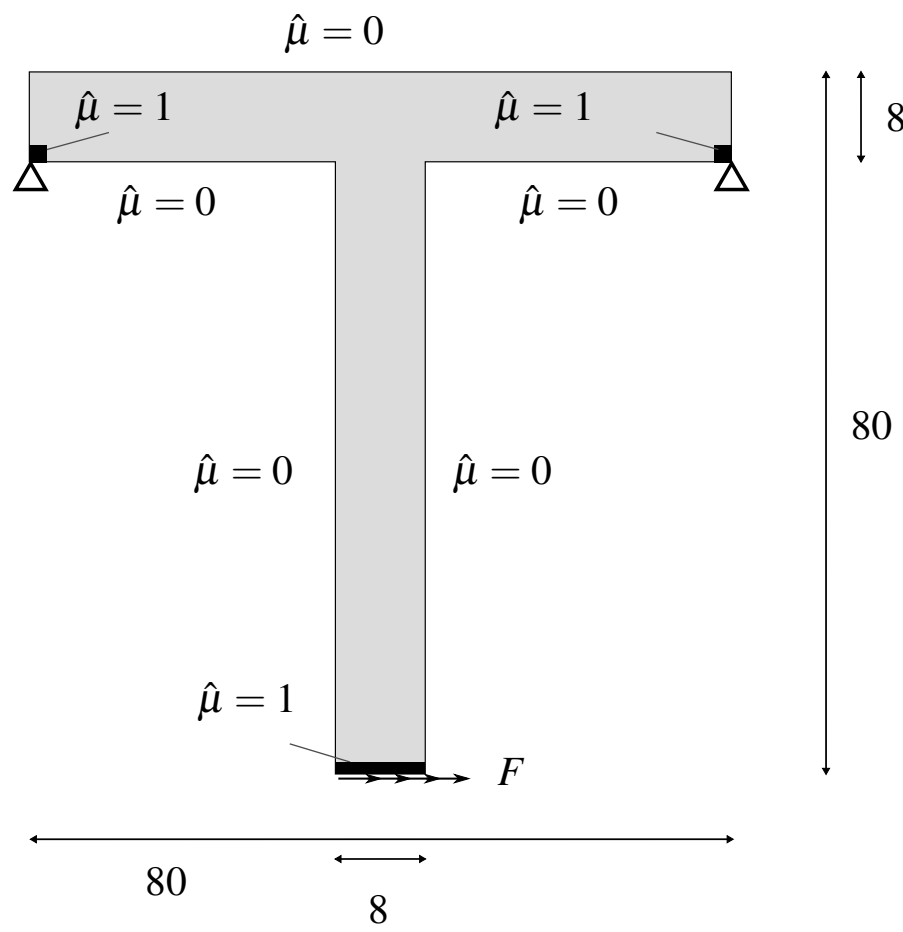

Figure 21: T-shaped design domain with two simple supports and a moment applied at the bottom. 


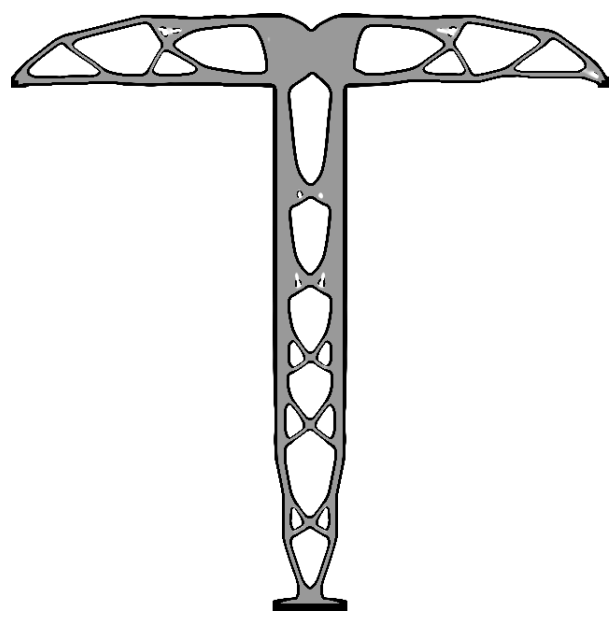

(a) $\lambda_{E}=0.40, c=4355$

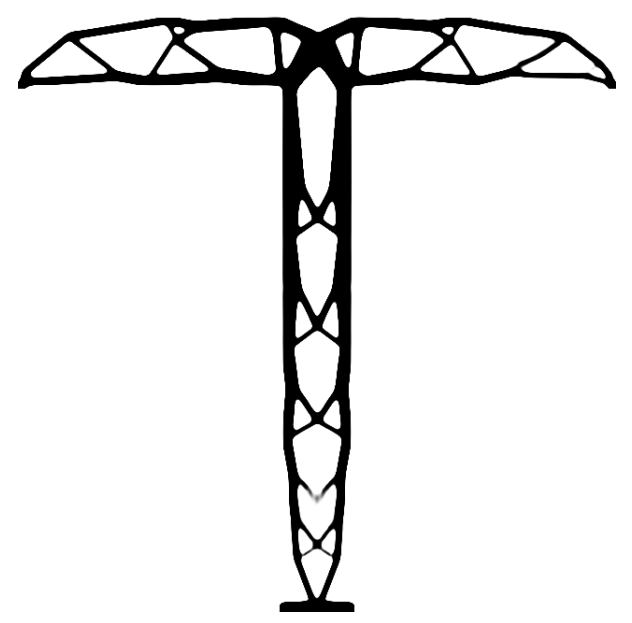

(b) Std. proj., $c=4012$

Figure 22: Optimized structures for T-domain shown in Fig 21

it does not allow the microstructure to adapt to differences in the principal stress directions. Even for the plate in bi-axial tension the porous infill leads to suboptimal designs. Microstructure is only advantageous when attributed sufficient design freedom. Including a buckling constraint this conclusion may change.

\section{Discussion}

This paper focuses on coated structures, however, several elements of the methodology are promising for broader applications. Particularly the two-step filtering process allowing to identify material interfaces and separate length scales of base structure and interface may be useful for problems involving interface modeling. An example is to include graded properties at the interface transition zone for multi-phase structures, thus suggesting an alternative, SIMP-based method to the level-set based approach suggested by Vermaak et al. (2014). The width and steepness of the interface may be modeled by modifying smoothing and projection parameters, or different interpolation schemes may be explored.

More generally speaking, applying the two-step filtering approach allows to crisply model material interfaces in SIMP-based topology optimization. Furthermore, two-step filtering approaches may find applications outside the area of interface modeling. A related double filtering concept is used to ensure robustness of topology optimized acoustic structures in Christiansen et al. (submitted). 
The study presented in this paper is limited to minimum compliance design. However, the primary objective of applying a coating is often to enhance other properties than stiffness. A range of alternative objective functions or physics problems could be considered. Examples of the latter include conduction or electromagnetic problems.

\section{Conclusions}

This paper introduces a new methodology for topology optimization of coated structures. The spatial gradient of the design field is used to identify material interfaces and apply the coating. A two-step filtering approach allows to control the gradient field and separate the length scales of the base structure and the coating. An analytical expression has been derived for the maximum coating thickness and the coating is shown to be applied with a highly uniform thickness over the structure.

The context of the paper is coating, however, several elements from the methodology are expected to be applicable to a broader range of problems. Especially the ability to identify and attribute particular properties to material interfaces is generally useful. In problems where only the length scale of the material interface is important one filtering step might be sufficient. Possible alternative applications have been discussed.

An alternative interpretation based on the typical additive manufacturing work flow has been presented. Rather than coating a base structure with a different material, the optimized structure is representing a single material. Objects optimized with this approach have a solid shell but a porous infill satisfying the HashinShtrikman bounds for isotropic material.

The effectiveness of the approach has been illustrated through a range of numerical results.

\section{Acknowledgments}

The authors would like to thank Erik Andreassen (DTU TopOpt group) for valuable discussions related to various topics of the paper. Furthermore, the introduction to coating technology provided by senior engineer at IPU's technology development, Peter Torben Tang, was much appreciated. The authors acknowledge financial support from the Villum Foundation (the NextTop project) and DTU Mechanical Engineering. 


\section{References}

Bendsøe, M. P., Sigmund, O., 1999. Material interpolation schemes in topology optimization. Archive of applied mechanics 69 (9-10), 635-654.

Bendsøe, M. P., Sigmund, O., 2003. Topology Optimization. Theory, Methods and Applications. Springer.

Borrvall, T., 2001. Topology optimization of elastic continua using restriction. Archives of Computational Methods in Engineering 8 (4), 351-385.

Christiansen, R. E., Lazarov, B. S., Jensen, J. S., Sigmund, O., submitted. Creating geometrically robust designs for acoustic cavity problems using topology optimization.

Guest, J., Prevost, J., Belytschko, T., 2004. Achieving minimum length scale in topology optimization using nodal design variables and projection functions. International Journal for Numerical Methods in Engineering 61 (2), 238-254.

Hashin, Z., Shtrikman, S., 1963. A variational approach to the theory of the elastic behaviour of multiphase materials. Journal of the Mechanics and Physics of Solids 11 (2), 127-140.

Lazarov, B. S., Sigmund, O., 2011. Filters in topology optimization based on helmholtz-type differential equations. International Journal for Numerical Methods in Engineering 86 (6), 765-781.

Møller, P., Nielsen, L., 2013. Advanced Surface Technology. Vol. 1-2. Møller \& Nielsen.

Petersson, J., Sigmund, O., 1998. Slope constrained topology optimization. International Journal for Numerical Methods in Engineering 41 (8), 1417-1434.

Schaedler, T., Jacobsen, A., Torrents, A., Sorensen, A., Lian, J., Greer, J., Valdevit, L., Carter, W., 2011. Ultralight metallic microlattices. Science 334 (6058), 962-965.

Sigmund, O., 2007. Morphology-based black and white filters for topology optimization. Structural and Multidisciplinary Optimization 33 (4-5), 401-424.

Sigmund, O., Maute, K., 2013. Topology optimization approaches. Structural and Multidisciplinary Optimization 48 (6), 1031-1055. 
Svanberg, K., 1987. Method of moving asymptotes - a new method for structural optimization. International Journal for Numerical Methods in Engineering 24 (2), 359-373.

Torquato, S., Gibiansky, L., Silva, M., Gibson, L., 1998. Effective mechanical and transport properties of cellular solids. International Journal of Mechanical Sciences 40 (1), 71-82.

van Dijk, N. P., Maute, K., Langelaar, M., Van Keulen, F., 2013. Level-set methods for structural topology optimization: a review. Structural and Multidisciplinary Optimization 48 (3), 437-472.

Vermaak, N., Michailidis, G., Parry, G., Estevez, R., Allaire, G., Bréchet, Y., 2014. Material interface effects on the topology optimization of multi-phase structures using a level set method. Structural and Multidisciplinary Optimization Published online, 1-22.

Wang, F., Lazarov, B. S., Sigmund, O., 2011. On projection methods, convergence and robust formulations in topology optimization. Structural and Multidisciplinary Optimization 43 (6), 767-784. 